\title{
The Volume Filling Factor of the WIM
}

\author{
Elly M. Berkhuijsen
}

Max-Planck-Institut für Radioastronomie, Auf dem Hügel 69, D-53121 Bonn, Germany

Abstract. A compilation of data on the volume filling factor $f_{\mathrm{v}}$ of the warm ionized medium (WIM) as a function of the local electron density $n_{\mathrm{e}}$ indicates that approximately $f_{v} \propto n_{\mathrm{e}}^{-1}$ over 4 decades. This result is expected for a fractal ISM.

\section{Introduction}

The volume filling factors of various constituents of the interstellar medium (ISM) are of great interest for the understanding of the physics of this medium (see Kulkarni and Heiles (1988), McKee (1995) and Cox (1995) for recent reviews). In this paper I present observational data on the volume filling factor of the warm ionized medium (WIM) obtained from the literature and by myself.

The volume filling factor is usually defined as

$$
f_{\mathrm{v}}=\frac{\left\langle n_{\mathrm{e}}\right\rangle}{n_{\mathrm{e}}}=\frac{\left\langle n_{\mathrm{e}}\right\rangle^{2}}{\left\langle n_{\mathrm{e}}^{2}\right\rangle}=\frac{\left\langle n_{\mathrm{e}}^{2}\right\rangle}{n_{\mathrm{e}}^{2}},
$$

where $n_{\mathrm{e}}$ is the local volume density of the WIM in $\mathrm{cm}^{-3}$ and brackets indicate averages. Pynzar (1993 and priv. comm.) has shown that for classical HII regions with $n_{\mathrm{e}} \gtrsim 100 \mathrm{~cm}^{-3} f_{\mathrm{v}}$ can be approximated by

$$
f_{\mathrm{v}} \simeq \frac{\text { diameter of HII region }}{\text { distance to HII region }} \text {. }
$$

The variables in (1a) can be found from the emission measure $\mathrm{EM}=\int n_{\mathrm{e}}^{2} d l$, the dispersion measure $\mathrm{DM}=\int n_{\mathrm{e}} d l$, and the rotation measure $\mathrm{RM}=$ $0.81 \int B_{\|} n_{\mathrm{e}} d l$, where the line of sight $l$ is in pc and the strength of the magnetic field along $l, B_{\|}$, in $\mu \mathrm{G}$. Then

$$
\begin{gathered}
n_{\mathrm{e}}=\mathrm{EM} / \mathrm{DM}, \\
\left\langle n_{\mathrm{e}}\right\rangle=\mathrm{DM} / L=\mathrm{RM} / 0.81 B_{\|} L, \\
\left\langle n_{\mathbf{e}}^{2}\right\rangle=\mathrm{EM} / L,
\end{gathered}
$$

where $L$ is the line of sight through the HII region considered. EM can be derived from observations of the $\mathrm{H} \alpha$ line, recombination lines and the thermal free-free radiation at radio wavelengths, DM from pulsar observations and RM from observations of pulsars or extragalactic radio sources. 


\section{Observations}

Figure 1 shows a compilation of the presently available data on $f_{\mathrm{v}}$ as a function of $n_{\mathrm{e}}$.

Cordes et al. (1991) constructed a model of the distribution of electrons in the Milky Way based on observations of EM, DM and the scattering measure SM. The model consists of 3 components of increasing density: I outer disk, II inner disk and III clumps, which have decreasing values of $f_{\mathrm{v}}$. Values of $n_{\mathrm{e}}$ for the clumps of $\sim 1$ pc size I took from Fig. 2 of Habing and Israel (1979).

Leahy (1987) analyzed the variations in RM between the lobes of radio galaxies. He argued that the observed variations must be due to irregularities in $n_{\mathrm{e}}$ of about $1 \mathrm{pc}$ rather than to variations in $B_{\|}$and gave the range of $f_{\mathrm{v}}$ and $n_{\mathrm{e}}$ shown by the box labelled L in Fig. 1. However, recently Minter and Spangler (1996) presented evidence that $B$ and $n_{\mathrm{e}}$ may be correlated making Leahy's estimate less certain.

Pynzar (1993) was the first who plotted $f_{v}$ as a function of $n_{\mathrm{e}}$. He used EM and DM towards pulsars and tried to separate interarm (P1 in Fig. 1) and arm (P2) regions along the line of sight using the spiral arm model of Georgelin and Georgelin (1976). For the classical HII regions (P3) he used EM and $f_{\mathrm{v}}$ from $(1 \mathrm{~b})$.

Heiles et al. (1996) derived $f_{\mathrm{v}}$ and $n_{\mathrm{e}}$ of the ELDWIM in a spiral arm region towards $l=20^{\circ}$ based on EM, DM and the model of the $n_{\mathrm{e}}$ distribution of Taylor and Cordes (1993).

Berkhuijsen (in prep.) calculated $f_{\mathrm{v}}$ by comparing the flux density of the thermal radio emission and that expected from the disk of thermal electrons as given by Reynolds (1991). A short description of this method is given in Berkhuijsen et al. (1997). The thermal radio emission at the solar radius $\left(R_{\odot}=8.5 \mathrm{kpc}\right)$ I calculated from the $408 \mathrm{MHz}$ model of Beuermann et al. (1985) and the spectral index map of Reich and Reich (1988), and I used an exponential fit of one $z$-component to the two $z$-components of the electron density given by Reynolds (1991). For the solar neighbourhood I find $f_{\mathrm{v}}=$ $0.038 \pm 0.010$ and $n_{\mathrm{e}}=\left\langle n_{\mathrm{e}}\right\rangle / f_{\mathrm{v}}=0.92 \mp 0.25 \mathrm{~cm}^{-3}$ at $z=0$. This value refers to a mixture of arm and interarm regions and is consistent with the results of Pynzar (1993) and Heiles et al. (1996).

\section{Results and Discussion}

In spite of the large uncertainties the data in Fig. 1 are fairly consistent with each other. The line connecting the points of Berkhuijsen and Heiles et al. (1996) has a slope of $-0.7 \pm 0.4$, in agreement with that of the line through the points in Pynzar's Fig. 2 of $-0.71 \pm 0.05$ (eye estimate). However, the results of Cordes et al. (1991) suggest a slope close to -1 . Thus $f_{\mathrm{v}}$ is approximately inversely proportional to $n_{\mathrm{e}}$. This means that $\left\langle n_{\mathrm{e}}\right\rangle=f_{\mathrm{v}} n_{\mathrm{e}} \simeq$ constant and about the same for regions of high and of low density. 


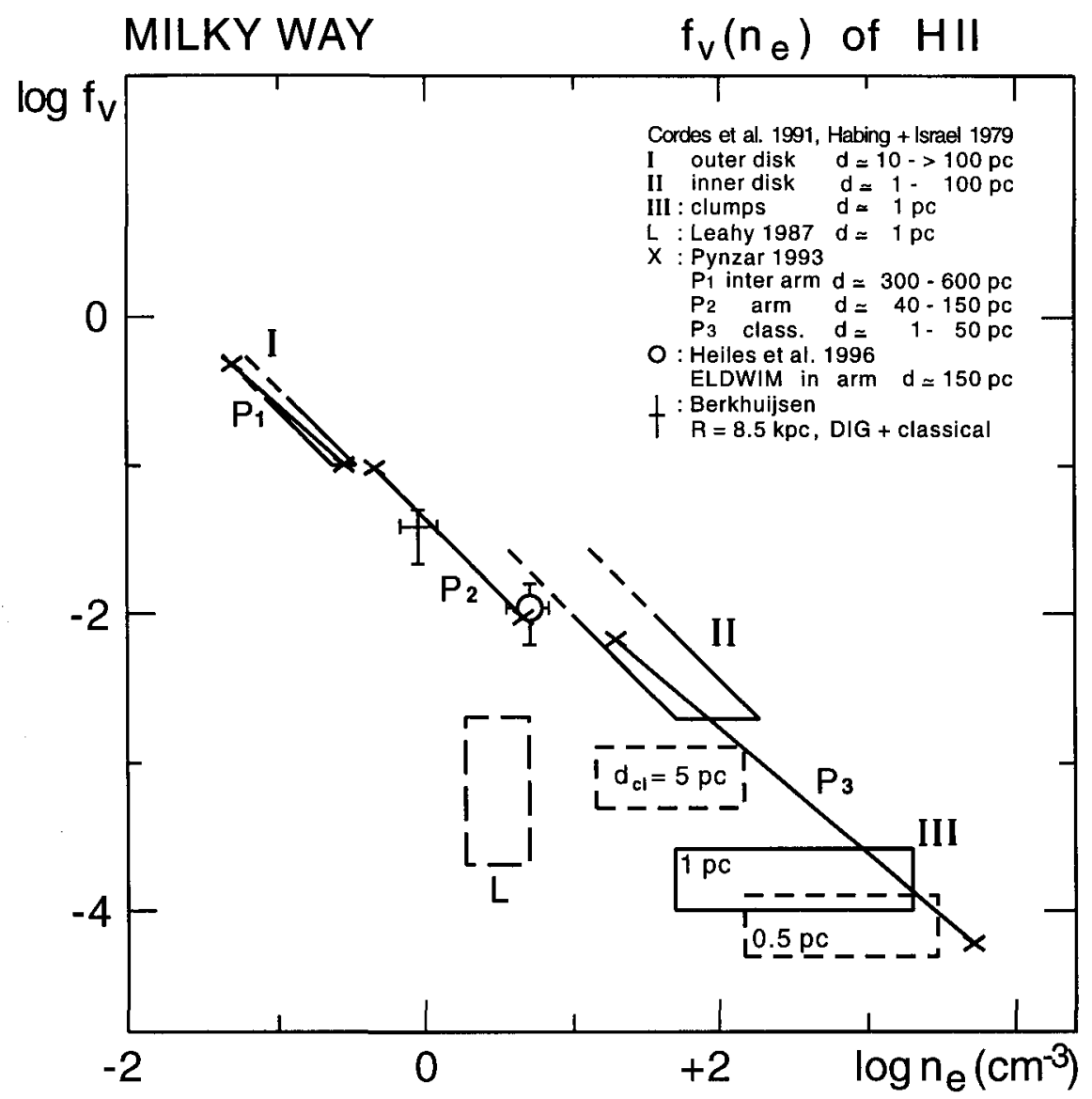

Fig. 1. The observed dependence of the volume filling factor $f_{\mathrm{v}}$ on the volume density of free electrons $n_{\mathrm{e}}$.

On inspection of Fig. 1 two problems arise:

a) $f_{\mathrm{v}}>1$ for $n_{\mathrm{e}} \lesssim 0.01 \mathrm{~cm}^{-3}$, thus no space is left for the hot interstellar medium (HIM). As the existence of the HIM seems well established $f_{\mathrm{v}}$ at low densities must be overestimated. This could happen if the electrons are distributed in sheets and filaments which occupy a smaller volume than spherical clouds. Sheets and filaments are indeed common in the ISM.

b) The filling factor of the total WIM, $f_{\mathrm{HII}}$, is the integral over $\log _{\mathrm{e}} n_{\mathrm{e}}$ of the function derived from Fig. 1. For the functions mentioned above integration yields $f_{\mathrm{HII}}=0.005$ for the classical HII regions $\left(100<n_{\mathrm{e}}<10^{4} \mathrm{~cm}^{-3}\right)$, but $f_{\mathrm{HII}}>1$ for the diffuse gas $\left(0.01<n_{\mathrm{e}}<100 \mathrm{~cm}^{-3}\right)$ which is impossible. This again shows that at low densities $f_{\mathrm{v}}$ is overestimated. On the other 
hand, simple integration of the distribution in Fig. 1 may lead to unrealistic values of $f_{\mathrm{HI}}$ if the values of $n_{\mathrm{e}}$ are averages themselves, i.e. referring to HII regions with substructure. And this generally is the case.

In spite of the problems indicated it is interesting that $f_{\mathrm{v}}$ is approximately inversely proportional to $n_{\mathrm{e}}$ as this is expected for a fractal ISM (Fleck 1996). Further evidence for a fractal structure of the ISM is the abundant existence of sheets, filaments and voids (Elmegreen 1997), and the observed slopes of the size distribution and the mass distribution of molecular clouds (Elmegreen and Falgarone 1996). If the WIM is predominantly fractal we may have to reconsider the usual derivations of $f_{\mathrm{v}}$ and $n_{\mathrm{e}}$ from observations of EM, DM and RM to avoid the problems sketched above.

In summary, the available data on $f_{\mathrm{v}}$ and $n_{\mathrm{e}}$ of the WIM show an approximately inverse relationship between these quantities, which is consistent with a fractal structure of the WIM. At low densities $f_{\mathrm{v}}$ appears to be overestimated. In order to improve the data on $f_{\mathrm{v}}$ a critical check of the derivation methods seems required.

\section{References}

Berkhuijsen, E.M., Horellou, C., Krause, M., Neininger, N., Poezd, A.D., Shukurov, A., Sokoloff, D.D. (1997): A\&A 318, 700

Beuermann, K., Kanbach, G., Berkhuijsen, E.M. (1985): A\&A 153, 17

Cordes, J.M., Weisberg, J.M., Frail, D.A., Spangler, S.R., Ryan, M. (1991): Nat 354,121

Cox, D.P. (1995): ASP Conference Ser. 80, 317

Elmegreen, B.G. (1997): ApJ 477, 196

Elmegreen, B.G., Falgarone, E. (1996): ApJ 471, 816

Fleck, R.C. (1996): ApJ 458, 739

Georgelin, Y.M., Georgelin, Y.P. (1976): A\&A 49, 57

Habing, H.J., Israel, F. (1979): ARA\&A 17, 345

Heiles, C., Reach, W.T., Koo, B-C. (1996): ApJ 466, 191

Kulkarni, S.R., Heiles, C. (1988): in Galactic and Extragalactic Radio Astronomy, eds. G.L. Verschuur, K.I. Kellermann, 2nd edition, p. 95

Leahy, J.P. (1987): MNRAS 226, 433

McKee, C.F. (1995): ASP Conference Ser. 80, 292

Minter, A.M., Spangler, S.R. (1996): ApJ 458, 194

Pynzar, A.V. (1993): Astron. Rep. 37, 245

Reich, P., Reich, W. (1988): A\&A 196, 211

Reynolds, R.J. (1991): Proc. IAU Symp. 144, p. 67

Taylor, J.H., Cordes, J.M. (1993): ApJ 411, 674 